Pacific Journal of 


\title{
COVERINGS OF A PROJECTIVE ALGEBRAIC MANIFOLD
}

\author{
KIYOSHI WATANABE
}

\begin{abstract}
Let $M$ be a projective algebraic manifold. Suppose $\pi: D \rightarrow M$ is a covering of $M$. If $D$ satisfies $H^{1}\left(D, O^{*}\right)=0$, then $D$ is a Stein manifold with $H^{2}(D, Z)=0$, where $O^{*}$ is the sheaf of germs of nowhere-vanishing holomorphic functions and $Z$ is the additive group of integers.
\end{abstract}

Let $D$ be a domain in $C^{n}$ and $\Gamma$ be a discrete subgroup of Aut $(D)$. It is well-known that if the quotient manifold $D / \Gamma$ is compact, then $D$ is a domain of holomorphy. Recently, CarlsonHarvey [1] showed that if $D$ is a domain in a Stein manifold and $D \rightarrow M$ is a covering of a compact Moisheson manifold $M$, then $D$ is a Stein manifold. On the other hand, we showed in [4] that if a pseudoconvex domain $D$ in a projective algebraic manifold satisfies $H^{1}\left(D, O^{*}\right)=0$, then $D$ is a Stein manifold with $H^{2}(D, Z)=0$.

In this paper, we study the case where a covering of a manifold is not contained in a larger manifold. We shall prove the following:

THEOREM. Let $M$ be a projective algebraic manifold. Suppose $\pi: D \rightarrow M$ is a covering of $M$. If $D$ satisfies $H^{1}\left(D, O^{*}\right)=0$, then $D$ is a Stein manifold with $H^{2}(D, Z)=0$.

We remark that the condition $H^{1}\left(D, O^{*}\right)=0$ cannot be replaced by $H^{1}(D, O)=0$, where $O$ is the sheaf of germs of holomorphic functions. To see this it is enough to consider the case $D=M=$ $P_{2}(C)$ and $\pi$ is the identity mapping.

Proof of theorem. Let $\left\{V_{i}\right\}$ be an open covering of $M$ such that each $V_{i}$ is a local coordinate neighborhood and is biholomorphic to a connected component $\pi^{-1}\left(V_{i}\right)$. Since $M$ is a projective algebraic manifold, there is a positive line bundle $F$ over $M$. Choosing a suitable refinement $\left\{U_{j}\right\}$ of $\left\{V_{i}\right\}$, we can represent $F$ by a system of transition functions $\left\{f_{j k}\right\}$ and find a Harmitian metric $\left\{a_{j}\right\}$ along the fibers of $F$ which satisfies the following conditions:

(i) Each $a_{j}$ is a $C^{\infty}$, real-valued and positive function on $U_{j}$,

(ii) If $U_{j} \cap U_{k} \neq \phi$, then we have $a_{k}=\left|f_{j k}\right|^{2} a_{j}$,

(iii) For every point $P$ in $M$, the Hessian of $-\log a_{j}$ relative to a local coordinate system $\left(z_{1}, \cdots, z_{n}\right)$ at $P$ 


$$
\begin{aligned}
L\left(-\log a_{j} ; P\right) & =\left(-\frac{\partial^{2} \log a_{j}}{\partial z_{\alpha} \partial \bar{z}_{\beta}}(P)\right) \\
(\alpha, \beta & =1, \cdots, n)
\end{aligned}
$$

is positive definite. By the compactness of $M, M$ has a finite open coverning $\left\{U_{j}: j=1, \cdots, m\right\}$.

Since $U_{j}$ is biholomorphic to each of the connected components of $\pi^{-1}\left(U_{j}\right)$, we have the functions $\left\{a_{j} \circ \pi\right\}$ which satisfies the following conditions:

(i ) Each $a_{j} \circ \pi$ is a $C^{\infty}$, real-valued and positive function on $\pi^{-1}\left(U_{j}\right)$,

(ii) If $\pi^{-1}\left(U_{j}\right) \cap \pi^{-1}\left(U_{k}\right) \neq \phi$, then we have $a_{j} \circ \pi=\left|f_{j k} \circ \pi\right|^{2} a_{k} \circ \pi$,

(iii) $W\left(-\log a_{j} \circ \pi ; P\right)$ is positive at every point $P$ in $D$, where

$$
W(\dot{\phi} ; P):=\min \left\{\sum_{\alpha, \beta} \frac{\partial^{2} \dot{\phi}}{\partial w_{\alpha} \partial \bar{w}_{\beta}}(P) \lambda_{\alpha} \bar{\lambda}_{\beta}: \sum_{\alpha}\left|\lambda_{\alpha}\right|^{2}=1, \quad \alpha, \beta=1, \cdots, n\right\}
$$

and $\left(w_{1}, \cdots, w_{n}\right)$ is a local coordinate at $P$.

Since $U=\left\{\pi^{-1}\left(U_{j}\right)\right\}$ is an open covering of $D,\left\{f_{j k} \circ \pi\right\}$ defines an element of $H^{1}\left(U, O^{*}\right)$. By the assumption of $H^{1}\left(D, O^{*}\right)=0$, there is a cochain $\left\{f_{j}\right\}$ of $C^{\circ}\left(U, O^{*}\right)$ such that $f_{j k} \circ \pi=f_{k} / f_{j}$. We can define a $C^{\infty}$ function $\phi$ on $D$ in the following way:

$$
\dot{\phi}(P):=-\log \left(a_{j} \circ \pi(P)\left|f_{j}(P)\right|^{2}\right)
$$

for $P$ in $\pi^{-1}\left(U_{j}\right)$. Since $M$ is paracompact, $M$ has a finite open covering $\left\{W_{j}: j=1, \cdots, m\right\}$ with $\bar{W}_{j} \subset U_{j}$. By the property (iii) there is a positive constant $C_{j}$ such that $W(\phi ; P)>C_{j}$ for $P$ in $\pi^{-1}\left(W_{j}\right)(j=1, \cdots, m)$. Hence we have

$$
W(\phi ; P)>C:=\min \left\{C_{j}: j=1, \cdots, m\right\}
$$

for $P$ in $D$. We remark that $D$ is not finitely sheeted, because $D$ has the strongly plurisubharmonic function $\phi$.

On the other hand, $M$ is a projective algebraic manifold, so $D$ has a real-analytic Kähler metric. Let $d(P, Q)$ be the distance between $P$ and $Q$ measured by the Kähler metric. Let us fix a point $P_{0}$ in $D$ and define a continuous function $\psi$ on $D$ in the following way:

$$
\psi(P):=d\left(P_{0}, P\right)
$$

for $P$ in $D$. We see that for every $c>0$, the set $\{P \in D: \psi(P)<c\}$ is relatively compact in $D$. Denotes by $\Gamma(P, \varepsilon)$ the set $\{Q \in D$ : $d(P, Q)<\varepsilon\}$, where a positive constant $\varepsilon$ is chosen so that $\pi(\Gamma(P, \varepsilon))$ 
is contained in some $U_{j}$ and $\Gamma(P, \varepsilon)$ is homeomorphic to a hypersphere. We define the following operator $A_{\varepsilon}$ mapping continuous function $f$ on $D$ into $C^{1}$ function on $D$ :

$$
A_{\varepsilon} f(P):=\frac{1}{V} \int_{-(P, \varepsilon)} f(Q) d v,
$$

where $d v$ is the volume element determined by the Kähler metric and $V$ is the volume of $\Gamma(P, \varepsilon)$. We see that the set $\left\{P \in D: A_{\varepsilon} \psi(P)<c\right\}$ is relatively compact in $D$. Let define

$$
\psi_{1}=A_{\varepsilon} \psi \text { and } \psi_{2}=A_{\varepsilon} \psi_{1}
$$

on $D$, then $\psi_{2}$ is $C^{2}$ and the set $\left\{P \in D: \psi_{2}(P)<c\right\}$ is also relatively compact in $D$. Let compute the Hessian of $\psi_{2}$. Since $D$ has a real-analytic Kähler metric, there are a local coordinate $\left(w_{1}, \cdots, w_{n}\right)$ of $\Gamma(P, \varepsilon)$ and a positive constant $K_{1}$ such that

$$
\left|\psi(Q)-\psi\left(Q^{\prime}\right)\right|^{2} \leqq K_{1}\left\{\left|w_{1}-w_{1}^{\prime}\right|^{2}+\cdots+\left|w_{n}-w_{n}^{\prime}\right|^{2}\right\}
$$

for two points $Q=\left(w_{1}, \cdots, w_{n}\right)$ and $Q^{\prime}=\left(w_{1}^{\prime}, \cdots, w_{n}^{\prime}\right)$ in $\Gamma(P, \varepsilon)$ (see [3] Lemma 1). By the compactness of $M, K_{1}$ can be chosen independent of $P$. Choosing $K_{1}$ large enough if necessary, we have

$$
\left|\frac{\partial \psi_{1}}{\partial w_{j}}(P)\right| \leqq K_{1} \quad(j=1, \cdots, n)
$$

and consequently

$$
\left|\frac{\partial^{2} \psi_{2}}{\partial w_{j} \partial \bar{w}_{k}}(P)\right| \leqq K_{1} \quad(j, k=1, \cdots, n)
$$

for $P$ in $D$. Therefore a positive constant $K$ can be chosen so that

$$
W\left(\psi_{2} ; P\right)>-K
$$

for $P$ in $D$. Now we define a $C^{2}$ function $\Phi$ on $D$ in the following way:

$$
\Phi(P):=K \cdot \phi(P)+C \cdot \psi_{2}(P)
$$

for $P$ in $D$. Then (1) and (2) induce

$$
W(\Phi ; P) \geqq K \cdot W(\phi ; P)+C \cdot W\left(\psi_{2} ; P\right)>0
$$

for $P$ in $D$. Hence $\Phi$ is a strongly plurisubharmonic function on $D$ and the set $\{P \in D: \Phi(P)<c\}$ is relatively compact in $D$ for every $c>0$. Therefore $D$ is a Stein manifold by Narasimhan [2]. Moreover from the exact sequence $0 \rightarrow Z \rightarrow O \rightarrow O^{*} \rightarrow 0$ we obtain the exact cohomology sequence 
$\cdots \longrightarrow H^{1}(D, O) \longrightarrow H^{1}\left(D, O^{*}\right) \longrightarrow H^{2}(D, Z) \longrightarrow H^{2}(D, O) \longrightarrow \cdots$

Since $H^{2}(D, O)=0$ by the Cartan's Theorem B and $H^{1}\left(D, O^{*}\right)=0$ by the assumption, we have $H^{2}(D, Z)=0$. This completes the proof.

\section{REFERENCES}

1. J. A. Carlson and R. Harvey, $A$ remark on the universal cover of a Moishezon space, Duke Math. J., 43 (1976), 497-500.

2. R. Narasimhan, The Levi problem for complex spaces II, Math. Ann., 146 (1962), 195-216.

3. A. Takeuchi, Domaines pseudoconvexes sur les variétés köhlériennes, J. Math. Kyoto Univ., 6 (1967), 323-357.

4. K. Watanabe, Cousin domains in an algebraic surface, Mem. Fac. Sci. Kyushu Univ., 29 (1975), 355-359.

Received December 20, 1978.

KOBE UNIVERSITY

NADA, KoBE, 657 JAPAN 


\section{PACIFIC JOURNAL OF MATHEMATICS}

\section{EDITORS}

DONALD BABBITT (Managing Editor)

University of California

Los Angeles, California 90024

Hugo RossI

University of Utah

Salt Lake City, UT 84112

C. C. MOORE and ANDREW OGG

University of California

Berkeley, CA 94720

\section{J. DugundJI}

Department of Mathematics University of Southern California Los Angeles, California 90007

R. FinN and J. Milgram Stanford University Stanford, California 94305

\section{ASSOCIATE EDITORS}
R. ARENS
E. F. BECKENBACH
B. H. Neumann
F. WOLF
K. YoshidA

\section{SUPPORTING INSTITUTIONS}

UNIVERSITY OF ARIZONA

UNIVERSITY OF BRITISH COLUMBIA

CALIFORNIA INSTITUTE OF TECHNOLOGY

UNIVERSITY OF CALIFORNIA

MONTANA STATE UNIVERSITY

UNIVERSITY OF NEVADA, RENO

NEW MEXICO STATE UNIVERSITY

OREGON STATE UNIVERSITY
UNIVERSITY OF OREGON

UNIVERSITY OF SOUTHERN CALIFORNIA

STANFORD UNIVERSITY

UNIVERSITY OF HAWAII

UNIVERSITY OF TOKYO

UNIVERSITY OF UTAH

WASHINGTON STATE UNIVERSITY

UNIVERSITY OF WASHINGTON 


\section{Pacific Journal of Mathematics}

\section{Vol. 96, No. $1 \quad$ November, 1981}

Hédi Amara, Groupe des classes et unité fondamentale des extensions quadratiques relatives à un corps quadratique imaginaire principal $\ldots \ldots \ldots 1$

Douglas S. Bridges, On the isolation of zeroes of an analytic function $\ldots \ldots 13$ Andrew J. Casson and John L. Harer, Some homology lens spaces which

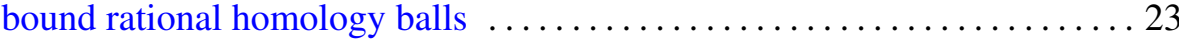

Z. A. Chanturia, On the absolute convergence of Fourier series of the classes $H^{\omega} \cap V[v]$

J.-F. Colombeau and Mário Carvalho Matos, On some spaces of entire functions defined on infinite-dimensional spaces $\ldots \ldots \ldots \ldots \ldots \ldots \ldots 63$

Edwin Duda, Pointwise periodic homeomorphisms on chainable continua . . .77

Richard F. Gustafson, A simple genus one knot with incompressible spanning surfaces of arbitrarily high genus $\ldots \ldots \ldots \ldots \ldots \ldots \ldots \ldots 1$

Fumio Hiai, Masanori Ohya and Makoto Tsukada, Sufficiency, KMS condition and relative entropy in von Neumann algebras

Ted Hurley, Intersections of terms of polycentral series of free groups and free Lie algebras. II .................................. 111

Robert Edward Jamison, II, Partition numbers for trees and ordered sets . . 115 R. D. Ketkar and N. Vanaja, A note on FR-perfect modules ............. 141 Michihiko Kikkawa, On Killing-Ricci forms of Lie triple algebras ....... 153

Jorge Lewowicz, Invariant manifolds for regular points 163

Richard W. Marsh, William H. Mills, Robert L. Ward, Howard Rumsey and Lloyd Richard Welch, Round trinomials .....

Claude Schochet, Topological methods for $C^{*}$-algebras. I. Spectral sequences

Yong Sian So, Polynomial near-fields?

Douglas Wayne Townsend, Imaginary values of meromorphic functions in the disk

Kiyoshi Watanabe, Coverings of a projective algebraic manifold .. 243

Martin Michael Zuckerman, Choosing $l$-element subsets of $n$-element sets 